\title{
Complete intravenous leiomyomatosis: a case report and literature review
}

\author{
Jian-Liang Wei ${ }^{1}$, Xiang Ji ${ }^{1}$, Peng Zhang ${ }^{1}$, Wen-Jing Chen ${ }^{1}$, Ya-Nan Zhao ${ }^{2 \#}$, Ming Liu ${ }^{2 \#}$ \\ ${ }^{1}$ Department of Cardiology, Affiliated Hospital of Shandong University of Traditional Chinese Medicine, Jinan, China; ${ }^{2}$ Department of Vascular \\ Surgery, Affiliated Hospital of Shandong University of Traditional Chinese Medicine, Jinan, China \\ \#These authors contributed equally to this work. \\ Correspondence to: Ya-Nan Zhao; Ming Liu. Department of Vascular Surgery, Affiliated Hospital of Shandong University of Traditional Chinese \\ Medicine, No. 16369 Jingshi Road, Jinan 250011, China. Email: morpheusunday@163.com; liuming404@163.com.
}

\begin{abstract}
Intravenous leiomyomatosis (IVL) is a relatively novel disease and can be aggressive. Since the first case was reported, it has a history of more than one hundred years, but the clinical incidence is extremely low, which has profound clinical research significance. Early recognition and management can prevent fatal consequences. IVL should be considered in female patients with a history of leiomyomas and intravascular filling defects. Because the patient's symptoms are not obvious, it is often misdiagnosed. We report on a female patient with IVL who presented with dizziness, and discuss the unexplained cause of filling defects within the right cardiac chambers and pulmonary arterial system. We present a complicated and rare case of IVL in a woman with a right atrial mass. A long, cord-like mass with inhomogeneous echo was detected by echocardiography. Coronary computed tomography (CT) showed a large hypodense mass occupying most of the right atrial cavity, spreading in both pulmonary arteries. Vascular ultrasound revealed a mixed echogenic mass invading the inferior vena cava like a rope. We performed surgery on her and complete resection on the tumor. We search for documents by searching databases such as National Center for Biotechnology Information (NCBI), China National Knowledge Infrastructure (CNKI) and Web of Science (WoS), etc. the recurrence rate recorded in the 4 cases series was $47.83 \%(11 / 23), 43.48 \%(20 / 46), 12.50 \%(2 / 16)$, and $10.00 \%(2 / 20)$, respectively.
\end{abstract}

Keywords: Intravenous leiomyomatosis (IVL); right atrial mass; surgery; case report; literature review

Submitted Sep 24, 2021. Accepted for publication Nov 10, 2021.

doi: 10.21037/apm-21-3093

View this article at: https://dx.doi.org/10.21037/apm-21-3093

\section{Introduction}

Intravenous leiomyomatosis (IVL) is a rare aggressive disease (1). Because the symptoms are not obvious, the disease is difficult to diagnose. The first case on IVL in 1896 (2) and Dürck (3) first reported on a patient with intracardiac leiomyomatosis (ICL) in 1907. Adult women are more susceptible to this disease $(4,5)$. Vascular ultrasonography is important for the diagnosis of IVL. The special point is that we found an anomaly pass the echocardiogram. To further confirm the diagnosis by computed tomography (CT) and vascular ultrasonography. Surgery is currently the most appropriate option for IVL and ICL (6). At present, radical tumor resection has been proven to be cured (7). The overall survival of patients at 3,5 and 10 years after operation was $87.5 \%, 75.0 \%$ and $56.3 \%$, respectively (8). Li et al. stated that the recurrence rate is almost 0 if complete resection is achieved $(9,10)$. We present the following article in accordance with the CARE reporting checklist (available at https://dx.doi.org/10.21037/ apm-21-3093).

\section{Case presentation}

A 55-year-old woman was admitted to our hospital because of dizziness over 3 months. She had a history of hypertension and coronary heart disease. She underwent a 


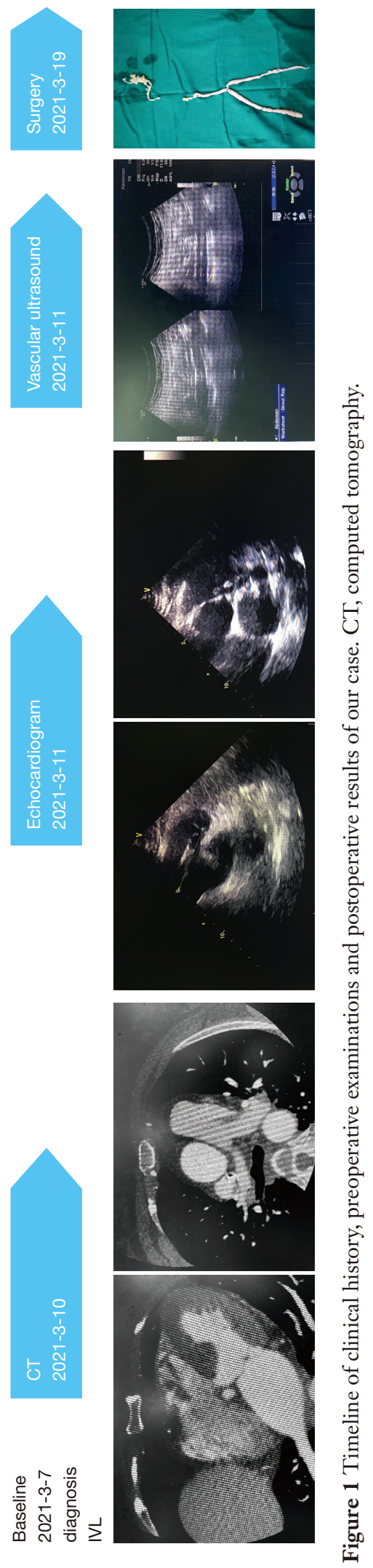

total abdominal hysterectomy 7 years earlier to treat uterine myoma. Baseline blood test on admission to the hospital showed hyperlipidemia.

A long, cord-like mass with inhomogeneous echo was detected through the right ventricular inflow and outflow tract and the 2 pulmonary arteries by echocardiography. Coronary CT showed a large low-density echogenic shadow in the right atrium, passing through the tricuspid valve to the right ventricle, and spreading in both pulmonary arteries. Vascular ultrasound revealed a solid, mixed echogenic mass invading the inferior vena cava like a rope (Figure 1).

All procedures performed in studies involving human participants were in accordance with the ethical standards of the institutional and/or national research committee(s) and with the Helsinki Declaration (as revised in 2013). Written informed consent was obtained from the patient for publication of this case report and accompanying images. A copy of the written consent is available for review by the editorial office of this journal.

\section{Discussion}

We searched the literature related to this disease in National Center for Biotechnology Information (NCBI), web of science (WoS), China national knowledge infrastructure (CNKI), etc. Birch-Hirschfeld first reported on IVL in 1896 (2). It is a rare benign neoplasm with quasi-malignant behavior characterized by intravascular smooth muscle cell proliferation $(2,9)$. IVL can be aggressive. As the tumor can extend into the inferior vena cava, the right cardiac chambers, and even the pulmonary artery, IVL can lead to vascular occlusion, heart blockage, or death $(9,11)$. In 1907, Dürck first reported on a patient with ICL (3).

Two views on the pathogenesis of IVL. One is that uterine leiomyomatosis invades the venous system and involves the inferior vena cava. The other is related to estrogen levels and chromosomal karyotypes. Changes in estrogen in the body lead to smooth muscle cells in the vein wall (12).

\section{Epidemiological characteristics}

The incidence of IVL is low. As of 2018, fewer than 300 cases of IVL patients have been reported (13). In recent years, the detection rate has gradually increased with diagnostic improvements, but case reports and small case series still dominate the literature. It has been reported 
that approximately $30 \%$ of women of reproductive age may suffer from this disease (14). Extrauterine involvement occurs in approximately $30 \%$ of IVL cases, and intracardiac extension accounts for about $10 \%(4,5)$.

Our literature review identified 18 articles and 245 patients with IVL in China. In our literature, $0.97 \%$ of patients with hysteromyoma were diagnosed with IVL (15), $54.3 \%$ of the IVL patients were intracardiac cases, and $13.5 \%$ involved the pulmonary artery. The mean detection age of IVL was at 50 years (11).

\section{Pathogenesis}

The pathogenesis of IVL is not fully understood. In 1975, Norris and Parmley reported that the origin of IVL might be from the wall of veins in the myometrium or develop due to the abnormally extensive vascular invasion of myometrial leiomyoma (16). Merchant et al. encountered a peculiar vascular structure in the myometrium, in which arteries were found to float freely in cleft-like spaces (17). By injecting different colored dyes into uterine arteries and veins, these spaces were shown to have venous channels and were considered important in the pathogenesis and development of IVL.

Quade et al. and Dal Cin et al. suggested that a specific chromosomal aberration, namely $\operatorname{der}(14) \mathrm{t}(12 ; 14)(\mathrm{q} 15 ; \mathrm{q} 24)$, might be a characteristic of IVL $(18,19)$. They proposed that IVL originated from a uterine leiomyoma with a $t(12 ; 14)(q 15 ; \mathrm{q} 24)$. The presence of an extra copy of 12q15qter and/or the loss of 14q24-qter may be a critical genetic event leading to intravascular intrusion and proliferation.

\section{Symptoms}

Almost $20-30 \%$ of IVL patients were asymptomatic. The common symptoms of IVL include pelvic mass, abnormal vaginal bleeding or excessive menstruation, abdominal distension or pain, chest distress or palpitation, lower limb swelling, dyspnea, or faintness. When the tumor spreads to the heart or pulmonary artery, more patients experience chest discomfort, shortness of breath, faintness, or sudden death $(4,20)$.

We report on a female IVL patient who presented with dizziness, and discuss the unexplained filling defects within the right cardiac chambers and pulmonary arterial system.

Li divided IVL into 4 stages according to the extent and course of the disease (21). In stage I there is almost no vascular obstruction or symptoms, except for lesions in the uterine. In stage II, the lesions invade the pelvic vessels, and there are usually no obvious clinical symptoms. In stage III, the tumor extends to the inferior vena cava, with clinical manifestations of symptoms, including lower extremity venous reflux disorder, inferior vena cava syndrome, or Budd-Chiari syndrome. In stage IV, the tumor invades the cardiac cavity, which might lead to right ventricular dysfunction.

\section{Medical bistory}

In our literature, the proportion of patients with uterine myoma in IVL was about $65.15 \%$, but in the 46 cases reported in Wang's study, all patients suffered from uterine myoma (22). A retrospective analysis showed that approximately $64 \%$ of patients had previously undergone hysterectomy, and the time from hysterectomy to the onset of IVL ranged from 6 months to 20 years (23). In our literature review, around $38.6 \%$ of patients had previously undergone myomectomy or hysterectomy.

\section{Transfer style}

IVL invades the systemic venous circulation mainly via the uterine iliac vein (the most common route) and the ovarian vein (20). Eighty-eight cases of metastasis were recorded, in which 70 were via the uterine iliac vein and the rest via the ovarian vein.

\section{Auxiliary examination}

\section{Vascular ultrasonography}

Vascular ultrasonography is important for the diagnosis of IVL and can provide information about intravascular thrombosis The main feature is a cord-like inhomogeneous substantive echo from the inferior vena cava extending to the right cardiac cavity with a clear boundary in the vessel and in the cardiac cavity (24).

Doppler ultrasound can be used to show the blood flow within the tumor thrombus. However, it is difficult to see small leiomyomas in small blood vessels, especially in asymptomatic patients.

Clement $e t$ al. divided IVL into 3 types according to the morphology and location of the tumor (25). The solid type is characterized by a large, solid hypoechoic mass in the paracervix or parauterine. The venous type is characterized 
by multiple bead-like nodules distributed along the uterine vein. The active type is characterized by a cord-like or worm-like tumor that enters the iliac vein.

\section{Echocardiography}

Echocardiography is typically chosen as the first-line method for ICL imaging. IVL should be suspected when echocardiogram reveals a right-sided cardiac mass originating from the inferior vena cava, especially in women with a history of hysterectomy or uterine leiomyoma. If the tumor has no stalk and moves freely in the inferior vena cava and right-sided cardiac chambers without attaching to the endothelial surface or endocardium, the diagnosis of IVL should be made (26).

\section{CT and magnetic resonance imaging (MRI)}

Imaging methods, such as CT or MRI, are generally used for IVL diagnosis due to high readability and large field of view. CT vascular reconstruction imaging can clearly show the tumor's location, size, and extension pathway, indicating its clinical significance in establishing a well-prepared surgical plan to achieve a good treatment outcome (27). After multiplanar reconstruction (MPR)/maximum intensity projection (MIP)/volume reconstruction (VR) or other $3 \mathrm{D}$ post-processing technologies of CT, the tumor can be displayed in all directions and from multiple angles. Tumors show different characteristics. Crutch-head sign in CT describes the shape of the tumor extending from the inferior vena cava to the right ventricular system. In addition, the tumor in the widened inferior vena cava was heterogeneously enhanced, and several cord-like tortuous blood vessels were observed within the tumor using CT. Zhang defined this as the hemp rope sign $(27,28)$.

MRI of an IVL will display isointense T1 and heterogeneous T2 signal intensity with heterogeneous postcontrast enhancement not seen in bland thrombi $(11,14)$.

\section{Pathology}

Pathology is the gold standard for diagnosis. Zhang found some patients' estrogen and progesterone receptors (PR) may be positive (29). In our literature, 128 of 133 patients recorded positive expression of estrogen. At the same time, 132 cases of progesterone expression and 51 cases of smooth muscle actin (SMA) expression were all positive.

Fan et al. found that desmin, SMA, and Ki-67 were positive in the ICL tumor, which could be used as biomarkers to identify IVL (30).

\section{Treatment}

\section{Surgery}

Surgery is the most common and effective tumor treatment option. One- or two-stage surgery can be selected based on a number of reasons, including the surgical tolerance of patients and the anatomical structure of the tumor $(31,32)$. The time of one-stage operation is generally longer, and the incidence of complications, such as bleeding, is higher. Two-stage surgery, which includes the suprarenal stage and infrarenal stage, is more likely to be used because of its shorter operation time, reduced risk of bleeding, and safer resection of intracardiac tumor masses (33). Guo et al. reported that the complications of one-stage surgery include pleural effusion, superior laryngeal nerve injury, and pulmonary infection, and the complications of two-stage surgery include pleural effusion, intestinal obstruction, and arrhythmia. There was no difference in the incidence of complications between the 2 operation styles. Two patients had tumors that progressed into the inferior vena cava when they underwent two-stage operation (34). Fan et al. found that the recurrence, complication, and infection rates in two-stage operation are lower than one-stage operation (30). However, Wang et al. found that, compared with one-stage surgery, two-stage surgery had a longer operation time, greater blood loss, and longer hospital stay (35).

A US study found that only $25 \%$ of patients had tumors completely removed during one-stage surgery, and $75 \%$ had residual lesions after surgery (36). Three perioperative deaths were due to intraoperative pulmonary artery rupture, intraoperative inferior vena cava laceration, and postoperative retroperitoneal hemorrhage. Ma et al. reported that cardiopulmonary bypass, especially deep hypothermic circulatory arrest, could be used as a surgical technique of patients with right atrial or even pulmonary artery invasion, reducing blood loss and successfully resecting tumors (37).

The successful treatment of IVL greatly depends on whether the tumor can be completely resected (38). Shen and $\mathrm{Ma}$ recommend bilateral salpingo-oophorectomy to avoid recurrence (39).

\section{Pharmacotherapy}

Considering the high estrogen receptor (ER) and PR expressions in IVL patients, hormonal therapy is widely used.

Kokawa et al. found that high estradiol and ER might affect the growth and invasion of IVL (40). In our literature, 
41 patients received hormonal drugs, such as tamoxifen or medroxyprogesterone. $\mathrm{Li}$ found that anti-estrogen therapy is also an important treatment for IVL patients with inoperable or residual tumors, or recurrence after surgery (21).

It has been reported that after 1 month of preoperative endocrine therapy (tamoxifen combined with goserelin acetate SR depot), when the condition improved, the tumor became smaller, and the staging operation could be performed (41). In their study, Guo et al. reported that 4 patients who received estrogen suppression therapy (triptorelin acetate or goserelin acetate + letrozole) before two-stage surgery had an increase in the size of their pelvic tumor, rather than a decrease (34).

There is little literature on whether hormone therapy can reduce the recurrence of IVL after complete tumor resection. The average treatment time was $6.83 \pm 0.66$ months. The patients were followed up for 3-55 months, and 6 patients relapsed (42).

It is uncertain whether hormone therapy can inhibit the growth of residual tumors in IVL patients with incomplete resection of tumors. Mitsuhashi et al. reported that a patient with IVL involving the atrium was treated with anti-estrogen for 6 months after incomplete resection of the tumor (43). After withdrawal anti-estrogen, the residual tumor in the pelvis increased significantly, and readministration continued to inhibit tumor growth. In their study, Hameleers et al. reported that a patient with IVL was treated with anti-estrogen for 3 years after incomplete removal of the tumor (44). Although the tumor increased slowly, it remained stable. In their literature review, Li et al. found that, in 36 cases with incomplete IVL resection, postoperative application of anti-estrogen therapy did not reduce the recurrence rate (10).

\section{Follow-up and recurrence}

We conducted the first telephone follow-up 6 months after the operation. The patient's vascular ultrasound examination at the local hospital showed that there was no obvious abnormal echo in the vein.

In our literature, the recurrence rate recorded in the 4 cases series was $47.83 \%(11 / 23), 43.48 \%(20 / 46)$, $12.50 \%(2 / 16)$, and $10.00 \%(2 / 20)$, respectively. Li et al. stated that the recurrence rate is almost 0 if complete resection is achieved (10); however, recurrence is expected in cases where complete resection is not achieved (35). In their study, Ahmed et al. reported a 30\% recurrence of postoperative tumors (45).
Wang et al. reported that the recurrence rate of IVL with inferior vena cava or heart involvement was $27.8 \%$ in their study (35).

Regardless of whether surgery is performed, long-term follow-up is essential. In previously published studies, some patients showed continued growth of incompletely resected intravenous tumors from 7 months to 15 years after surgery, resulting in a high recurrence rate of $30 \%$ (46). Du et al. reported that the recurrence rate of 18 patients with IVL their 2011 study was $16.6 \%$, suggesting that the young age of onset and the initial size of the tumor may be predisposing factors for recurrence (47).

The recommended follow-up intervals are 3, 6, and 12 months after operation, and annually after that (30). Pelvic ultrasound, venous ultrasound, and CT venogram follow-up is at the clinician's discretion, and there are currently no guidelines (30).

In conclusion, IVL is a rare disease that often occurs in perimenopausal women with symptoms of venous thrombosis or right atrial mass. Early detection and accurate diagnosis is a necessary condition for good recovery. Surgery is still the best treatment option for IVL.

\section{Acknowledgments}

Funding: The present study was supported by the National Natural Science Foundation of China (No. 82004369) and National Clinical Research Base of TCM (No. 2008-23).

\section{Footnote}

Reporting Checklist: The authors have completed the CARE reporting checklist. Available at https://dx.doi. org/10.21037/apm-21-3093

Conflicts of Interest: All authors have completed the ICMJE uniform disclosure form (available at https://dx.doi. org/10.21037/apm-21-3093). The authors have no conflicts of interest to declare.

Ethical Statement: The authors are accountable for all aspects of the work in ensuring that questions related to the accuracy or integrity of any part of the work are appropriately investigated and resolved. All procedures performed in studies involving human participants were in accordance with the ethical standards of the institutional and/or national research committee(s) and with the Helsinki Declaration (as revised in 2013). Written informed consent 
was obtained from the patient for publication of this case report and accompanying images. A copy of the written consent is available for review by the editorial office of this journal.

Open Access Statement: This is an Open Access article distributed in accordance with the Creative Commons Attribution-NonCommercial-NoDerivs 4.0 International License (CC BY-NC-ND 4.0), which permits the noncommercial replication and distribution of the article with the strict proviso that no changes or edits are made and the original work is properly cited (including links to both the formal publication through the relevant DOI and the license). See: https://creativecommons.org/licenses/by-nc-nd/4.0/.

\section{References}

1. Madhavan S, Junnarkar SP, Koh NWC, et al. Inferior vena cava leiomyosarcoma in an octogenerian. Ann Hepatobiliary Pancreat Surg 2019;23:274-7.

2. Birch-Hirschfeld FV. Lehrbuch der pathologischen anatomie. 5th edition. Leipzig: FCW Vogel, 1896.

3. Dürck H. Ueber ein kontinuierlich durch die untere Hohlvene indas Herz vorwachsendes Fibromyom des Uterus. Muench Med Wochenschr 1907;23:1154.

4. Castelli P, Caronno R, Piffaretti G, et al. Intravenous uterine leiomyomatosis with right heart extension: successful two-stage surgical removal. Ann Vasc Surg 2006;20:405-7.

5. Baca López FM, Martínez-Enriquez A, Castrejón-Aivar FJ, et al. Echocardiographic study of an intravenous leiomyoma: case report and review of the literature. Echocardiography 2003;20:723-5.

6. Liu J, Liang M, Ma G, et al. Surgical treatment for intravenous-cardiac leiomyomatosis. Eur J Cardiothorac Surg 2018;54:483-90.

7. Teixeira FJR Jr, do Couto Netto SD, Perina ALF, et al. Leiomyosarcoma of the inferior vena cava: Survival rate following radical resection. Oncol Lett 2017;14:3909-16.

8. Jeong S, Han Y, Cho YP, et al. Clinical Outcomes of Surgical Resection for Leiomyosarcoma of the Inferior Vena Cava. Ann Vasc Surg 2019;61:377-83.

9. Xu ZF, Yong F, Chen YY, et al. Uterine intravenous leiomyomatosis with cardiac extension: Imaging characteristics and literature review. World J Clin Oncol 2013;4:25-8.

10. Li B, Chen X, Chu YD, et al. Intracardiac leiomyomatosis: a comprehensive analysis of 194 cases. Interact Cardiovasc
Thorac Surg 2013;17:132-8.

11. Shi T, Shkrum MJ. A Case Report of Sudden Death From Intracardiac Leiomyomatosis. Am J Forensic Med Pathol 2018;39:119-22.

12. Wang L, Yang $\mathrm{Y}, \mathrm{Li} M \mathrm{MH}$, et al. Intravenous leiomyomatosis of uterus: report of a case. Zhonghua Bing Li Xue Za Zhi 2019;48:648-50.

13. Demirkiran F, Sal V, Kaya U, et al. Intravenous leiomyoma with extension to the heart: a case report and review of the literature. Case Rep Obstet Gynecol 2013;2013:602407.

14. Gunderson CC, Parsons B, Penaroza S, et al. Intravenous leiomyomatosis disguised as a large deep vein thrombosis. J Radiol Case Rep 2016;10:29-35.

15. Chen J. Intravenous leiomyomatosis: a study of 20 cases and literature review [ $\mathrm{PhD}$ thesis]. Shandong University, 2019.

16. Norris HJ, Parmley T. Mesenchymal tumors of the uterus. V. Intravenous leiomyomatosis. A clinical and pathologic study of 14 cases. Cancer 1975;36:2164-78.

17. Merchant S, Malpica A, Deavers MT, et al. Vessels within vessels in the myometrium. Am J Surg Pathol 2002;26:232-6.

18. Quade BJ, Dal Cin P, Neskey DM, et al. Intravenous leiomyomatosis: molecular and cytogenetic analysis of a case. Mod Pathol 2002;15:351-6.

19. Dal Cin P, Quade BJ, Neskey DM, et al. Intravenous leiomyomatosis is characterized by a $\operatorname{der}(14) \mathrm{t}(12 ; 14)$ (q15;q24). Genes Chromosomes Cancer 2003;36:205-6.

20. Lam PM, Lo KW, Yu MY, et al. Intravenous leiomyomatosis: two cases with different routes of tumor extension. J Vasc Surg 2004;39:465-9.

21. Li CM. Clinical characteristics and research progress of inferior vena cava leiomyomatosis. Chinese Journal of Vascular Surgery (Electronic Version) 2015;7:198-201.

22. Wang TT. Clinicopathological features and molecular mechanism of intravenous leiomyomatosis [ $\mathrm{PhD}$ thesis]. Peking Union Medical College, 2015.

23. Harris LM, Karakousis CP. Intravenous leiomyomatosis with cardiac extension: tumor thrombectomy through an abdominal approach. J Vasc Surg 2000;31:1046-51.

24. Polizzi V, Pergolini A, Zampi G, et al. Intravenous leiomyomatosis extending to the heart: a multimodality imaging approach. Herz 2014;39:720-1.

25. Clement PB, Young RH, Scully RE. Intravenous leiomyomatosis of the uterus. A clinicopathological analysis of 16 cases with unusual histologic features. Am J Surg Pathol 1988;12:932-45.

26. Li R, Shen Y, Sun Y, et al. Intravenous leiomyomatosis 
with intracardiac extension: echocardiographic study and literature review. Tex Heart Inst J 2014;41:502-6.

27. Zhang S, Zhang Q, Gong M, et al. Value of CT combined with ultrasonography in diagnosis of uterine intravenous leiomyomatosis invading the right heart system. Journal of Medical Imaging 2019;29:2055-8.

28. Chen X, Zhang XL, Ma XJ, et al. Clinical application of multi-detector row CT in the diagnosis of intravenous leiomyomatosis. Radiologic Practice 2013;28:784-7.

29. Zhang T, Zhang XM, Zhang XM, et al. Intravenous leiomyomatosis with intracardiac extension: a case report. Beijing Da Xue Xue Bao Yi Xue Ban 2008;40:642-4.

30. Fan JX, Zhou J, Wang YR, et al. Analysis of the diagnosis and treatment of uterine venous smooth muscle tumour involving the heart. Journal of Cardiovascular and Pulmonary Diseases 2019;38:274-8

31. Butany J, Singh G, Henry J, et al. Vascular Smooth MuscleTumors: 13 cases and a review of the literature. Int J Angiol 2006;15:43-50.

32. Ma G, Miao Q, Liu X, et al. Different surgical strategies of patients with intravenous leiomyomatosis. Medicine (Baltimore) 2016;95:e4902.

33. Okada M, Miyoshi Y, Kato G, et al. Successful onestage surgical removal of intravenous leiomyomatosis with cardiac extension in an elderly patient. Gen Thorac Cardiovasc Surg 2012;60:153-6.

34. Guo LL, Diao YP, Lian LS, et al. Therapeutic strategy of intravenous leiomyomatosis with intracaval and intracardiac extension in 18 Cases. Chinese Journal of Bases and Clinics in General Surgery 2014;21:335-40.

35. Wang J, Yang J, Huang H, et al. Management of intravenous leiomyomatosis with intracaval and intracardiac extension. Obstet Gynecol 2012;120:1400-6.

36. Worley MJ Jr, Aelion A, Caputo TA, et al. Intravenous leiomyomatosis with intracardiac extension: a singleinstitution experience. Am J Obstet Gynecol 2009;201:574.e1-5.

37. Ma GT, Miao Q, Liu XR, et al. Surgical Treatment Strategies of Intravenous Leiomyomatosis withRight
Cardiac Cavities Extension. Zhongguo Yi Xue Ke Xue Yuan Xue Bao 2016;38:438-43.

38. Murphy AN, Byrne D, Salati U, et al. Intravenous leiomyomatosis manifesting as saddle embolism. BMJ Case Rep 2019;12:228267.

39. Shen J, Ma J. Advances of diagnosis and treatment of intravenous leiomyomatosis with cardiac extension. Chinese Journal of Clinical Thoracic and Cardiovascular Surgery 2011;18:249-52.

40. Kokawa K, Yamoto M, Yata C, et al. Postmenopausal intravenous leiomyomatosis with high levels of estradiol and estrogen receptor. Obstet Gynecol 2002;100:1124-6.

41. Xu YC, Ye M, Sun L, et al. One case report of intravenous leiomyomatosis with right atrium extension. Journal of Shanghai Jiaotong University (Medical Science) 2011;31:866-8.

42. Yu X, Zhang GR, Shi H, et al. Clinical features and prognosis of intravenous leiomyomatosis with intracardiac extension. Journal of Reproductive Medicine 2016;25:483-6.

43. Mitsuhashi A, Nagai Y, Sugita M, et al. GnRH agonist for intravenous leiomyomatosis with cardiac extension. A case report. J Reprod Med 1999;44:883-6.

44. Hameleers JA, Zeebregts CJ, Hamerlijnck RP, et al. Combined surgical and medical approach to intravenous leiomyomatosis with cardiac extension. Acta Chir Belg 1999;99:92-4.

45. Ahmed M, Zangos S, Bechstein WO, et al. Intravenous leiomyomatosis. Eur Radiol 2004;14:1316-7.

46. Moniaga NC, Randall LM. Uterine leiomyomatosis with intracaval and intracardiac extension. Gynecol Oncol Case Rep 2012;2:130-2.

47. Du J, Zhao X, Guo D, et al. Intravenous leiomyomatosis of the uterus: a clinicopathologic study of 18 cases, with emphasis on early diagnosis and appropriate treatment strategies. Hum Pathol 2011;42:1240-6.

(English Language Editor: R. Scott)
Cite this article as: Wei JL, Ji X, Zhang P, Chen WJ, Zhao YN, Liu M. Complete intravenous leiomyomatosis: a case report and literature review. Ann Palliat Med 2021;10(11):12039-12045. doi: 10.21037/apm-21-3093 